\title{
Use of Initial Noninvasive Cardiac Testing and Association with Downstream Healthcare Resource: A Retrospective Review of Resources Used Across a 12-Month Window for Probable Type I Myocardial Infarction Patients
}

\author{
Dana Villines $^{1} \cdot$ Wm. Thomas Summerfelt ${ }^{1,2} \cdot$ James R. Spalding $^{3} \cdot$ Therese M. Kitt $^{3} \cdot$ Rita M. Kristy $^{3} \cdot$ Christy R. Houle $^{3}$
}

Published online: 17 May 2019

(c) The Author(s) 2019

\begin{abstract}
Background Clinical guidelines provide clinicians with substantial discretion in the use of noninvasive cardiac testing for patients with suspected coronary artery disease. Repeat testing, frequent emergency department (ED) visits, and increases in other cardiac-related procedures can be a burden on patients and payers and can complicate treatment planning. We assessed downstream healthcare resource utilization (HCRU) for patients undergoing initial single-photon emission computed tomography (SPECT), myocardial perfusion imaging (MPI), stress echocardiography (ECHO), or exercise treadmill testing (ETT) with probable type I myocardial infarction (MI). Methods Electronic medical records data from 12,130 patients with probable type I MI presenting to EDs within a large healthcare system comprised of 11 adult hospitals were retrospectively analyzed. Logistic and linear regression determined the individual contribution of SPECT-MPI, ETT, and ECHO on repeat cardiovascular (CV) testing, inpatient visits, outpatient visits, and cardiac-related costs within 12 months of the index visit.

Results The majority of patients received SPECT-MPI for the index-testing event (56.5\%), followed by ETT (29.2\%) and ECHO (14.3\%). Patients who had SPECT-MPI at the index visit were less likely to have a repeat CV testing visit (odds ratio [OR] 0.77, 95\% confidence interval [CI] $0.62-0.96 ; p=0.020)$ or an inpatient visit (OR 0.70, 95\% CI 0.49-0.98; $p=0.039)$ than those who underwent ETT or ECHO. ETT and ECHO were not predictive of any outcome.

Conclusions SPECT-MPI does not result in more downstream HCRU than ETT or ECHO and is associated with a lower likelihood of repeat non-invasive $\mathrm{CV}$ testing and inpatient visits.
\end{abstract}

\section{Key Points for Decision Makers}

Single-photon emission computed tomography myocardial perfusion imaging (SPECT-MPI) may initially cost more than exercise treadmill testing or echocardiography, but is associated with fewer downstream healthcare resource utilization visits.

SPECT-MPI was associated with fewer inpatient visits. SPECT-MPI was associated with less repeat cardiovascular testing.

Electronic supplementary material The online version of this article (https://doi.org/10.1007/s41669-019-0128-1) contains supplementary material, which is available to authorized users.

Dana Villines

Dana.Villines@advocatehealth.com

1 Advocate Health Care, 814 W. Nelson St, RM2025, Chicago, IL 60657, USA

\section{Introduction}

Cardiovascular (CV) disease continues to represent a significant and increasing cost burden in the US. Total direct costs (based on annual medical spending, in the form of insurer and out-of-pocket payments) were estimated at $\$ 273$ billion for 2010 and are expected to triple by 2030 , to $\$ 818$ billion [1]. Physicians can choose from a number of strategies for cardiac stress testing, a noninvasive diagnostic tool in the evaluation and management of patients with known or suspected coronary heart disease. Testing strategies include exercise treadmill testing (ETT) and several non-invasive CV imaging techniques, including echocardiography (ECHO), computed tomography angiography

\footnotetext{
2 Present Address: Convergence CT, Chicago, IL, USA

Astellas Pharma Global Development, Northbrook, IL, USA
} 
(CTA), and single-photon emission computed tomography (SPECT) myocardial perfusion imaging (MPI) [2].

Although diagnostic workup decisions for patients presenting with $\mathrm{CV}$ symptoms such as chest pain are primarily based on physician assessment and consideration of evidence-based clinical practice guidelines [3], growing cost-containment pressure may limit availability and access to certain higher-cost testing procedures. High initial testing costs may be offset by reductions in retesting procedures and emergency department (ED) visits. Overall, while many studies have addressed the diagnostic capabilities and subsequent clinical outcomes associated with CV diagnostic procedures [4-8], less is known about downstream healthcare resource utilization (HCRU, related to repeated testing and ED visits as well as inpatient and outpatient care) and costs related to the various non-invasive testing modalities, with few studies and inconsistent results for any given procedure (e.g. SPECT-MPI [9-12]).

The primary objectives of this study were to examine the downstream cardiac-related HCRU and costs associated with non-invasive testing utilization in the evaluation of patients with probable type I myocardial infarction (MI). Type I MI is distinguished from type II MI based on a variety of clinical factors, including the cause of ischemia/injury; type I is cardiac injury-related to atherosclerotic plaque rupture, ulceration, fissuring, erosion or dissection, while type II is cardiac injury due to an imbalance between myocardial oxygen supply and/or demand [13]. HCRU outcomes included repeat non-invasive testing, repeat ED visit, inpatient visits, outpatient visits, and cardiac-related costs within 12 months of the index visit.

\section{Methods}

In this retrospective, observational study, an electronic medical records (EMR) database was queried for a set of prespecified variables to assess the clinical and economic outcomes associated with non-invasive testing. Patientlevel clinical data, CV testing modality, and HCRU data were abstracted from the EMR database of a large healthcare system comprised of 11 adult hospitals with a designated Heart Institute including over 100 outpatient facilities and 350 heart specialists. The healthcare system is a not-for-profit entity with an approximate payer mix as follows: $45 \%$ managed care, $32 \%$ Medicare, $15 \%$ Medicaid, and $8 \%$ workers compensation, self-pay or other. Data were initially identified during the abstraction (to match files) and then de-identified. Institutional Review Board (IRB) approval from the hospitals was obtained prior to data collection (\#6290; Advocate Health Care IRB); patient informed consent was not required.

All aspects of the study design were developed by a steering committee comprised of cardiology physicians and physician leadership, a cardiology nurse, pharmacists, cardiology coding and billing experts, and research department leadership. Working groups were established within the committee to advise on specific protocol items, such as the research questions, endpoints, and measurement of endpoints. Working groups presented their recommendations to the steering committee for discussion and revision as appropriate.

\subsection{Study Sample and Inclusion/Exclusion Criteria}

The study sample included adult patients ( $\geq 18$ years of age) presenting to the ED for CV-related issues between 1 January 2009 and 31 July 2014 who had not had a previous ED visit, hospitalization, or non-invasive $\mathrm{CV}$ testing event within the prior 30 days of the initial (index) non-invasive $\mathrm{CV}$ testing event. Using Current Procedural Terminology (CPT) codes associated with SPECT-MPI, ECHO, ETT, and CTA, 91,769 patients were initially identified. Only patients presenting with probable type I MI with no evidence of type II MI based on primary and secondary admission diagnosis codes were included in the analysis, resulting in a potential sample of 15,772 patients (Fig. 1). After removing ineligible patients, 12,130 patients were included in the final study sample (Fig. 1).

\subsection{Variable Definition and Measurement}

Variable identification was via specific International Classification of Diseases, Ninth Revision (ICD-9) or CPT codes (see electronic supplementary index for codes) and linked hospital data (downstream visits, procedures, and diagnostic information associated with the codes identified). The ED admission and index date was defined as the first ED admission seen within the study timeframe meeting the inclusion/ exclusion criteria, including the first non-invasive $\mathrm{CV}$ testing seen within 7 days after the index date. Downstream HCRU outcome variables were defined as repeat non-invasive $\mathrm{CV}$ testing, inpatient visits, outpatient visits, and cardiac-related costs. Repeat non-invasive CV testing (not restricted to the same testing as at index), ED visit, and inpatient and outpatient visit outcomes were defined as subsequent cardiacrelated visits to the respective location post index date. ICD-9 codes were first used to identify cases of MI, followed by the use of CPT codes to identify testing modalities. Utilization was counted as the number of visits within the 12 months post index visit, but was dichotomized to yes (at least one visit)/ 
Fig. 1 Sample selection. $C V$ cardiovascular, $E D$ emergency department. Subjects with the following procedures (primary or secondary admission diagnosis) were excluded on the basis of being surgical or medical emergencies where imaging would not be undertaken preoperatively: aortic dissection, pulmonary embolism, gastrointestinal ulcer with hemorrhage and/or perforation, and acute myocardial infarction

$$
\mathbf{N}=\mathbf{9 1 , 7 6 9}
$$

Patients who presented to the ED and had not had a previous ED visit, hospitalization, or non-invasive $\mathrm{CV}$ testing event within the prior 30 days of the initial (index) non-invasive $\mathrm{CV}$ testing event

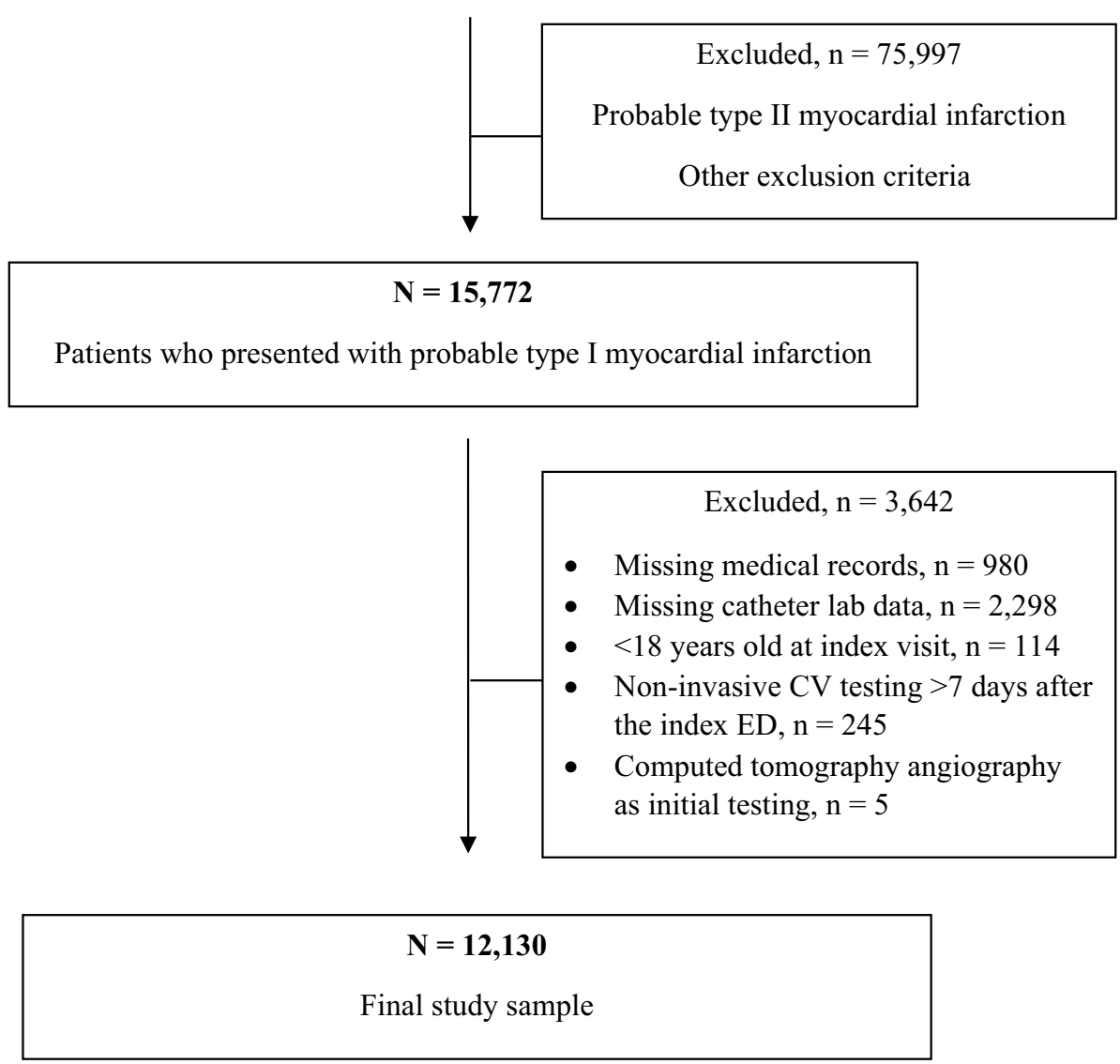

no (zero visits) after data collection due to the majority of patients having either zero or one visit. Cardiac-related costs and visits were broadly defined as any cardiac imaging and cardiac-related intervention within 12 months from the index event. An internal audit report was reviewed by the working group including cardiologists, cardiology coding, and billing experts to verify appropriate codes for cardiac procedures.

Additional variables included in the analysis were demographic, clinical, and provider-related. Demographic variables were age at index visit (analyzed as a continuous variable), sex, and race (Caucasian vs. non-Caucasian), while clinical variables were diabetes diagnosis, current smoker, and length of stay (LOS; analyzed as a continuous variable) at index visit and risk group as defined by the index visit discharge status. Provider-related variables included insurance/payer (private, government, or none), admission source (clinic, transfer, or directly to the ED), hospital type (academic or non-academic), and physician type (cardiologist or other). Although all patients were initially seen in the
$\mathrm{ED}$, the admission source could have been recorded as clinic (arrived at the ED from an outpatient clinic), ED (arrived at the ED independently), or transfer (transfer from another facility, such as a long-term-care nursing facility).

Academic hospitals were defined as those that have residency and fellowship training programs at the respective institution, which included 3 of the 11 hospitals. Physician type was based on the physician who ordered the non-invasive CV testing procedure at the index visit. Estimated costs were calculated by averaging per-unit costs in US dollars across the system and applying those per-unit costs to each procedure and encounter. Costs were estimated by the institution's Finance Department and represented an average per-unit cost across the system for procedures by factoring in personnel time, maintenance, depreciation, and overheads. This approach was chosen over reimbursement rates (charges), which can vary widely based on the population served and hospital location. 


\subsection{Comparative Groups}

Data stratification occurred for two variables-risk and testing modality. Risk groups were identified as a potential covariate and were defined as low risk (outpatient) and high risk (inpatient), depending on the patient's discharge status from the index ED visit. More specifically, patients who were either managed in observation status and discharged, or were discharged from the ED with follow-up visits as outpatients, were defined as low risk, while patients admitted to the hospital directly from the ED, or managed in the observation unit and then admitted, were defined as high risk.

Non-invasive CV testing groups for analysis were planned as follows: ETT - treadmill test with no additional testing (including no concurrent imaging modality) during the index visit; SPECT-MPI test—nuclear stress test; stress ECHOstress echocardiogram; and CTA.

\subsection{Data Validation and Analysis}

Data validation was performed prior to any analysis. Code and cross-reference data validation occurred whenever possible. For example, modality imaging data were abstracted by CPT code and by modality name to compare abstraction. Data were abstracted from multiple sources (data warehouse and quality department data abstraction) and compared for accuracy. Range and constraint validation occurred for all downstream HCRU variables.

Prior to analysis for the primary outcomes, descriptive and between-group analyses were performed, with noninvasive $\mathrm{CV}$ testing modalities as the grouping variable. Between-group differences were determined using Pearson's Chi square analysis for categorical variables and analysis of variance (ANOVA) for continuous variables.

To evaluate the association between downstream HCRU and the specified non-invasive CV testing modalities, multivariate linear and logistic regression was performed. Repeat non-invasive $\mathrm{CV}$ testing, repeat $\mathrm{ED}$ visit, and inpatient and outpatient visit outcomes were evaluated using logistic regression, while the cardiac-related cost outcome was evaluated using linear regression. Covariates to be included in the multivariate linear or logistic regression models were selected based on the results of univariate regression analyses performed to determine the independent contribution of each potential covariate. Results of linear regression are presented as beta coefficients $(\beta)$ with $95 \%$ confidence intervals (CIs), and logistic regression is presented as odds ratios (OR) with $95 \%$ CIs. Covariates were included in the multivariate model if the univariate evaluation resulted in a $p$ value $\leq 0.05$. All multivariate regression models included the same potential covariates (defined in Sect. 2.2): age (continuous), sex, race, diabetes diagnosis, current smoker, LOS at index visit (continuous), risk group, insurance, admission source, hospital type, and physician type. Age and LOS coefficients are interpreted as each unit increase above the mean for the predictors (i.e. 1 year older or 1 day longer in LOS) corresponds to an increase in the outcome by the coefficient while holding all other covariates constant.

Statistical significance was determined at $p \leq 0.05$, and all hypothesis testing was two-tailed. Analyses were performed using SPSS $22.0^{\circledR}$ for Windows (IBM Corporation, Armonk, NY, USA).

\section{Results}

\subsection{Demographic, Clinical, and Provider-Related Variable Distributions}

Overall, 12,130 patients were identified as meeting the inclusion/exclusion criteria (after excluding five patients who were identified as having CTA as their initial non-invasive CV testing, given the small sample size). Demographic clinical and provider-related variables are presented in Table 1, including the distribution across the non-invasive CV testing groups. The majority of patients received SPECT-MPI for the index non-invasive CV testing event (56.5\%), followed by ETT $(29.2 \%)$ and ECHO (14.3\%). The mean age of the sample was $64.9 \pm 13.79$ years. Half of the sample $(50.5 \%)$ was Caucasian and $40.4 \%$ were male. Diabetes diagnosis was reported by $36.2 \%$ of the sample, while current smoker was reported by $16.3 \%$. Admission to the ED from a clinic setting was the most frequent admission source (70.6\%), and academic hospitals were the site of care for $44.8 \%$ of the sample. Approximately half of the patients $(52.0 \%)$ were admitted to the hospital (academic or non-academic) from the ED and were therefore categorized as high risk. Non-invasive procedures were ordered by a cardiologist for $50.8 \%$ of patients. Mean LOS for the index visit was $2.2 \pm 2.53$ days, with $12.1 \%$ of patients referred to the catheterization laboratory and only $0.3 \%$ of patients readmitted within 30 days.

While statistically significant differences were found between the non-invasive $\mathrm{CV}$ testing groups for all of the demographic, clinical, and provider-related variables, the between-group differences were $<10 \%$ for most variables. Differences $>10 \%$ between groups were found for race, risk group, hospital type and physician type, and are detailed in Table 1.

\subsection{Downstream Healthcare Resource Utilization Outcomes with Univariate Predictors}

Occurrence rates for the outcomes of interest are displayed in Table 1, with the corresponding distribution across 
Table 1 Between-group analyses of demographic, clinical, and provider-related variables

\begin{tabular}{|c|c|c|c|c|c|}
\hline & Total sample $[n=12,130]$ & ECHO $[n=1733]$ & $\operatorname{ETT}[n=3545]$ & SPECT-MPI $[n=6852]$ & $p$ value \\
\hline Age at index, years $($ mean $\pm S D)$ & $64.9 \pm 13.79$ & $61.2 \pm 13.60$ & $66.5 \pm 13.43$ & $64.9 \pm 13.85$ & $<0.001$ \\
\hline Sex [male] & $4904(40.4)$ & $588(33.9)$ & $1432(40.4)$ & $2884(42.1)$ & $<0.001$ \\
\hline Race [Caucasian] & $6124(50.5)$ & $602(34.8)$ & $1481(41.8)$ & $4041(59.1)$ & $<0.001$ \\
\hline Diabetes diagnosis & $4386(36.2)$ & $597(34.4)$ & $1393(39.3)$ & $2396(35.0)$ & $<0.001$ \\
\hline Current smoker & $1978(16.3)$ & $350(20.2)$ & $562(15.9)$ & $1066(15.6)$ & $<0.001$ \\
\hline Admit source [clinic] & $8562(70.6)$ & $1270(73.3)$ & $2419(68.2)$ & $4873(71.1)$ & 0.004 \\
\hline Academic hospital & $5439(44.8)$ & $1052(60.7)$ & $2532(71.4)$ & $1855(27.1)$ & $<0.001$ \\
\hline Risk [high risk] & $6302(52.0)$ & $691(39.9)$ & $2238(63.1)$ & $3373(49.2)$ & $<0.001$ \\
\hline Physician [cardiologist] & $6156(50.8)$ & $685(39.5)$ & $1950(55.0)$ & $3521(51.4)$ & $<0.001$ \\
\hline Private insurance & $3938(32.5)$ & $631(40.0)$ & $1068(31.9)$ & $2239(35.1)$ & $<0.001$ \\
\hline Government insurance & $7261(59.9)$ & $919(58.3)$ & $2226(66.5)$ & $4116(64.5)$ & $<0.001$ \\
\hline LOS at index visit (mean \pm SD) & $2.2 \pm 2.53$ & $1.9 \pm 2.05$ & $2.6 \pm 2.88$ & $2.1 \pm 2.42$ & $<0.001$ \\
\hline Catheter laboratory referral & $1473(12.1)$ & $133(7.7)$ & $501(14.1)$ & $839(12.2)$ & $<0.001$ \\
\hline Readmit within 30 days & $40(0.3)$ & $8(0.5)$ & $11(0.3)$ & $21(0.3)$ & 0.585 \\
\hline Repeat CV testing & $1277(10.5)$ & $199(11.5)$ & $504(14.2)$ & $574(8.4)$ & $<0.001$ \\
\hline ED revisit & $78(0.2)$ & $7(0.4)$ & $8(0.2)$ & $13(0.2)$ & 0.251 \\
\hline Inpatient visit & $601(5.0)$ & $85(4.9)$ & $290(8.2)$ & $226(3.3)$ & $<0.001$ \\
\hline Outpatient visit & $647(5.3)$ & $112(6.5)$ & $208(5.9)$ & $327(4.8)$ & 0.005 \\
\hline $\operatorname{Cost}^{\mathrm{a}}($ mean $\pm \mathrm{SD})$ & $965.2 \pm 2092.3$ & $659.7 \pm 1855.1$ & $927.3 \pm 1987.9$ & $1104.5 \pm 2244.5$ & 0.031 \\
\hline
\end{tabular}

Data are expressed as $n(\%)$ unless otherwise specified

$C V$ cardiovascular, ED Emergency Department, ECHO echocardiography, ETT exercise treadmill testing, LOS length of stay, $S D$ standard deviation, SPECT-MPI single-photon emission computed tomography myocardial perfusion imaging

${ }^{\mathrm{a}}$ Cost $=$ downstream costs (in US\$) for cases with an ED, outpatient, and/or inpatient revisit

the non-invasive $\mathrm{CV}$ testing groups. Repeat non-invasive $\mathrm{CV}$ testing within 12 months of the index visit occurred for $10.5 \%$ of patients, irrespective of the testing modality at index visit. While $5.0 \%$ and $5.3 \%$ of patients had an inpatient and outpatient visit post index visit, respectively, very few patients $(0.2 \%)$ had a return visit to the ED within 12 months. The mean downstream cost for patients who had any return visit within 12 months of the index visit was $\$ 965.2 \pm 2092.3$. ETT had the most repeat non-invasive CV testing (14.2\%) and inpatient visits (8.2\%), while ECHO had the most outpatient visits (6.5\%) and MPI had the highest mean cost $(\$ 1104.5 \pm 2244.5)$. Between-group differences for repeat ED visits were not statistically significant. Furthermore, none of the potential covariates were associated with repeat ED visits at a statistically significant level (data not shown), therefore further analysis was not performed for this outcome.

ETT, ECHO, and SPECT-MPI, as well as potential covariates, were assessed via univariate regression (Table 2). $\mathrm{ECHO}$ was not associated with repeat non-invasive CV testing within 12 months of the index visit, while ETT was associated with increased repeat testing (OR 1.68, 95\% CI 1.49-1.89) and SPECT-MPI was less likely to have repeat testing (OR 0.60, 95\% CI 0.53-0.67). SPECT-MPI also was less likely to have an inpatient visit within 12 months of the index visit (OR $0.45,95 \%$ CI $0.38-0.53$ ), while ETT was associated with an increased risk of inpatient visit (OR 2.37, 95\% CI 2.01-2.80) and no association was observed for ECHO. ECHO was associated with an increased risk of an outpatient visit (OR 1.27, 95\% CI 1.03-1.57) but SPECTMPI was less likely to have an outpatient visit (OR 0.78, 95\% CI 0.66-0.91) and ETT was not associated with outpatient visits. Finally, ETT was associated with higher downstream cardiac-related costs ( $\beta=42.70,95 \%$ CI 13.73-71.67), while SPECT-MPI and ECHO were not associated at a statistically significant level $(\beta=-20.89,95 \% \mathrm{CI}-47.48$ to 5.69 ; $\beta=-30.18,95 \% \mathrm{CI}-67.84$ to 7.48 , respectively).

\subsection{Multivariate Predictors}

The covariates identified as relevant to the multivariate models were similar across the tested models but were not identical (Table 2; statistically significant variables, indicated by '*' and '**', were included in the corresponding multivariate analysis.). Male sex and Caucasian race were associated with repeat non-invasive $\mathrm{CV}$ testing, inpatient visits, and increased cardiac-related cost; increasing age was associated with fewer inpatient and outpatient visits. Diabetes diagnosis, admission to an academic hospital, and 
Table 2 Univariate logistic and linear regression parameters for downstream healthcare utilization and costs

\begin{tabular}{|c|c|c|c|c|}
\hline & $\begin{array}{l}\text { Repeat CV testing } \\
{[\text { OR }(95 \% \text { CI })]}\end{array}$ & Inpatient visit [OR $(95 \% \mathrm{CI})]$ & Outpatient visit [OR (95\% CI)] & $\begin{array}{l}\text { Cardiac-related cost }[\beta \text { coef- } \\
\text { ficient }(95 \% \mathrm{CI})]\end{array}$ \\
\hline ETT at index & $1.68(1.49-1.89)^{* *}$ & $2.37(2.01-2.80)^{* *}$ & $1.16(0.98-1.37)$ & $42.70(13.73-71.67)^{* *}$ \\
\hline MPI at index & $0.60(0.53-0.67)^{* *}$ & $0.45(0.38-0.53)^{* *}$ & $0.78(0.66-0.91)^{* *}$ & $-20.89(-47.48$ to 5.69$)$ \\
\hline ECHO at index & $1.12(0.96-1.32)$ & $0.99(0.78-1.25)$ & $1.27(1.03-1.57)^{*}$ & $-30.18(-67.84$ to 7.48$)$ \\
\hline Age at index & $1.00(1.00-1.00)$ & $1.01(1.00-1.02)^{* *}$ & $0.99(0.99-1.00)^{* *}$ & $-0.18(-1.13$ to 0.78$)$ \\
\hline Sex [male] & $1.16(1.03-1.31)^{*}$ & $1.31(1.11-1.55)^{* *}$ & $1.03(0.88-1.21)$ & $47.25(20.41-74.10)^{* *}$ \\
\hline Caucasian & $1.14(1.02-1.28)^{*}$ & $1.19(1.01-1.40)^{*}$ & $1.07(0.91-1.25)$ & $51.19(24.78-77.59)^{* *}$ \\
\hline Clinic admit & $0.89(0.70-1.13)$ & $0.59(0.44-0.79)^{* *}$ & $1.48(1.01-2.15)^{*}$ & -22.07 ( -73.98 to 29.84$)$ \\
\hline ED admit & $1.35(0.98-1.84)$ & $2.40(1.67-3.45)^{* *}$ & $0.53(0.29-0.97)^{*}$ & $17.60(-56.21$ to 91.40$)$ \\
\hline Transfer admit & $0.92(0.65-1.29)$ & $1.05(0.66-1.68)$ & $0.84(0.52-1.34)$ & $24.26(-45.68$ to 94.20$)$ \\
\hline High risk & $1.20(1.07-1.35)^{* *}$ & $2.67(2.22-3.21)^{* *}$ & $0.57(0.49-0.67)^{* *}$ & $48.50(22.13-74.86)^{* *}$ \\
\hline Diabetes diagnosis & $1.52(1.35-1.71)^{* *}$ & $1.72(1.46-2.02)^{* *}$ & $1.23(1.05-1.45)^{*}$ & $54.92(27.50-82.33)^{* *}$ \\
\hline Current smoker & $0.92(0.79-1.08)$ & $0.99(0.79-1.23)$ & $0.88(0.70-1.10)$ & $-3.68(-39.36$ to 31.99$)$ \\
\hline Academic site & $1.60(1.43-1.80)^{* *}$ & $1.95(1.65-2.31)^{* *}$ & $1.32(1.12-1.54)^{* *}$ & $39.39(12.90-65.88)^{* *}$ \\
\hline Physician (cardiologist) & $0.85(0.76-0.96)^{* *}$ & $1.04(0.89-1.23)$ & $0.76(0.65-0.89)^{* *}$ & $7.54(-18.82$ to 33.90$)$ \\
\hline LOS at index visit & $1.04(1.02-1.06)^{* *}$ & $1.09(1.06-1.11)^{* *}$ & $0.94(0.90-0.98)^{* *}$ & $29.64(24.45-34.82)^{* *}$ \\
\hline Private insurance & $0.78(0.68-0.89)^{* *}$ & $0.54(0.45-0.66)^{* *}$ & $1.07(0.90-1.27)$ & $-13.36(-41.88$ to 15.16$)$ \\
\hline Government insurance & $1.27(1.12-1.45)^{* *}$ & $1.79(1.47-2.17)^{* *}$ & $0.93(0.79-1.11)$ & $16.32(-12.02$ to 44.67$)$ \\
\hline No insurance & $1.19(0.67-2.14)$ & $1.36(0.63-2.95)$ & $1.07(0.47-2.46)$ & $-78.00(-219.64$ to 63.64$)$ \\
\hline
\end{tabular}

$O R$ odds ratio, $C I$ confidence interval, $C V$ cardiovascular, $E D$ emergency department, $E C H O$ echocardiography, ETT exercise treadmill testing, $L O S$ length of stay, MPI myocardial perfusion imaging

$* p \leq 0.05, * * p \leq 0.01$

longer LOS at index visit were associated with increased risk for all outcomes, and longer LOS at index visit was associated with less likelihood of an outpatient visit. High-risk patients were more likely to have repeat non-invasive CV testing and inpatient visits, and less likely to have outpatient visits. Increased risk for repeat non-invasive $\mathrm{CV}$ testing and inpatient visits was associated with government insurance. Testing procedure ordered by a cardiologist was associated with a decreased likelihood of repeat non-invasive CV testing and outpatient visits.

Similar variables were predictive of the four outcomes assessed in the multivariate model as in the univariate analysis. All predictors of the outcomes, as ORs or $\beta$ with $95 \%$ CIs, are displayed in Table 3. SPECT-MPI was the only noninvasive $\mathrm{CV}$ testing that remained predictive in the multivariate models. Patients who had SPECT-MPI at the index visit were less likely to have repeat non-invasive $\mathrm{CV}$ testing (OR $0.77,95 \%$ CI $0.62-0.96 ; p=0.020)$ and less likely to have an inpatient visit (OR 0.70, 95\% CI 0.49-0.98; $p=0.039$ ). ETT and ECHO were not predictive of any outcome and none of the non-invasive procedures were associated with outpatient revisits or with costs in the multivariate model.

In addition to SPECT-MPI, cardiologist as the ordering physician was associated with a decreased likelihood of repeat non-invasive $\mathrm{CV}$ testing. An increased likelihood of repeat non-invasive $\mathrm{CV}$ testing was associated with
Caucasian race, diabetes diagnosis, academic hospital, and LOS at index visit. MPI was the only protective factor, indicating a reduced risk, for inpatient visits. However, similar predictors for inpatient visits were found as for repeat noninvasive $\mathrm{CV}$ testing, i.e. Caucasian race, high risk, diabetes diagnosis, academic hospital, and LOS at index visit.

In general, variables that indicated patients are at risk for repeat non-invasive testing were also indicators that patients are at risk for more inpatient visits. Statistically significant covariates for outpatient visits and cardiac-related costs were similar to the predictors previously listed. All of the statistically significant predictors of cost were associated with increased costs as follows: male sex, Caucasian race, diabetes diagnosis, and LOS at index visit.

\section{Discussion}

In this analysis of 12,130 probable type I MI patients who visited an $\mathrm{ED}$ with subsequent $\mathrm{CV}$ testing procedures performed, four outcomes were assessed within 12 months of the index event: repeat non-invasive $\mathrm{CV}$ testing, repeat $\mathrm{ED}$ visits, and inpatient and outpatient visits. SPECT-MPI at the index visit was associated with a lower likelihood of having an outpatient visit or repeat non-invasive CV testing. ETT and ECHO were not predictive of any outcome. 
Table 3 Multivariate logistic and linear regression parameters for downstream healthcare utilization and costs

\begin{tabular}{lllll}
\hline & $\begin{array}{l}\text { Repeat CV testing [OR } \\
(95 \% \mathrm{CI})], p \text { value }\end{array}$ & $\begin{array}{l}\text { Inpatient visit [OR }(95 \% \\
\mathrm{CI})], p \text { value }\end{array}$ & $\begin{array}{l}\text { Outpatient visit [OR }(95 \% \\
\mathrm{CI})], p \text { value }\end{array}$ & $\begin{array}{l}\text { Cardiac-related cost }[\beta \text { coef- } \\
\text { ficient }(95 \% \mathrm{CI})], p \text { value }\end{array}$ \\
\hline ETT at index & $1.09(0.87-1.36), 0.441$ & $1.36(0.98-1.88), 0.066$ & $-(-)-$ & $28.86(-2.34$ to 60.05$), 0.070$ \\
MPI at index & $0.77(0.62-0.96), 0.020$ & $0.70(0.49-0.98), 0.039$ & $1.03(0.82-1.29), 0.824$ & $-(-)-$ \\
ECHO at index & $-(-)-$ & $-(-)-$ & $1.19(0.90-1.57), 0.223$ & $-(-)-$ \\
Age at index & $-(-)-$ & $0.99(0.99-1.00), 0.186$ & $0.99(0.99-1.00), 0.024$ & $-(-)-$ \\
Sex [male] & $1.14(0.99-1.32), 0.076$ & $1.19(0.96-1.47), 0.107$ & $-(-)-$ & $35.45(8.53-62.37), 0.010$ \\
Caucasian & $1.25(1.08-1.46), 0.003$ & $1.28(1.02-1.59), 0.031$ & $-(-)-$ & $54.21(27.53-80.88),<0.001$ \\
Clinic admit & $-(-)-$ & $0.95(0.58-1.56), 0.853$ & $1.24(0.77-1.99), 0.372$ & $-(-)-$ \\
ED admit & $1.01(0.73-1.39), 0.971$ & $1.35(0.74-2.47), 0.333$ & $0.67(0.31-1.44), 0.307$ & $-(-)-$ \\
Transfer admit & $-(-)-$ & $-(-)-$ & $-(-)-$ & $-(-)-$ \\
High risk & $1.07(0.92-1.25), 0.392$ & $1.78(1.40-2.25),<0.001$ & $0.72(0.59-0.88), 0.001$ & $-7.91(-36.04$ to 20.23), 0.582 \\
Diabetes diagnosis & $1.50(1.29-1.73), 0.000$ & $1.56(1.26-1.93),<0.001$ & $1.37(1.139-1.648), 0.001$ & $43.15(15.38-70.93), 0.002$ \\
Current smoker & $-(-)-$ & $-(-)-$ & $-(-)-$ & $-(-)-$ \\
Academic site & $1.23(1.05-1.44), 0.012$ & $1.33(1.05-1.68), 0.020$ & $1.25(1.03-1.53), 0.028$ & $14.52(-13.71$ to 42.74), 0.313 \\
Physician (cardiologist) & $0.77(0.66-0.89), 0.001$ & $-(-)-$ & $0.89(0.73-1.07), 0.218$ & $-(-)-$ \\
LOS at index visit & $1.04(1.02-1.07), 0.002$ & $1.06(1.03-1.09),<0.001$ & $1.00(0.95-1.04), 0.941$ & $28.10(22.59-33.62),<0.001$ \\
Private insurance & $0.78(0.40-1.54), 0.482$ & $0.50(0.21-1.19), 0.117$ & $-(-)-$ & $-(-)-$ \\
Government insurance & $0.94(0.48-1.85), 0.863$ & $0.82(0.34-1.93), 0.642$ & $-(-)-$ & $-(-)-$ \\
No insurance & $-(-)-$ & $-(-)-$ & $-(-)-$ & $-(-)-$ \\
\hline
\end{tabular}

Variables included in the univariate analysis that were not statistically significant are not included in the corresponding multivariate model and are indicated by $-(-)-$

Model $R^{2}$ : Repeat CV Testing $=0.01$, Inpatient Visit $=0.03$, Outpatient Visit $=0.02$, Cardiac-Related Cost $=0.07$

$O R$ odds ratio, $C I$ confidence interval, $C V$ cardiovascular, $E D$ emergency department, $E C H O$ echocardiography, ETT exercise treadmill testing, $L O S$ length of stay, MPI myocardial perfusion imaging

While SPECT-MPI was associated with fewer inpatient visits and a lower likelihood of repeat non-invasive CV testing, and was not predictive of higher cardiac-related costs (any subsequent cardiac charge after the index visit), the mean cardiac-related cost was higher for this testing group than ETT and ECHO (approximate difference was $\$ 180$ and $\$ 445$, respectively).

Many of the demographic, clinical, and provider-related variables were associated with increased downstream HCRU. As expected, clinical factors played a large role. As patients with dual diagnosis are, in general, more likely to have exacerbated healthcare needs compared with patients with a single medical complication, it is not surprising that patients with diabetes are more likely to have an inpatient and/or outpatient visit, repeat non-invasive CV testing, and more cardiac-related costs. Similarly, longer LOS at index visit was associated with repeat non-invasive CV testing, inpatient visits and increased costs, but the relationship was weak for all three outcomes. This may be an artifact of sicker patients requiring longer care and more follow-up care than patients who were quickly discharged from the ED or index inpatient visit. Patients with a longer LOS at index visit are likely to be sicker patients requiring longer care and more follow-up care than patients who were quickly discharged from the $\mathrm{ED}$ or index inpatient visit.
In this study, the subjects tended to be a similar age to other published studies [4-6] and approximately $10 \%$ more females were included in the sample than in other studies. In this study, almost twice the rate of diabetes and less than half the rate of smokers were reported compared with other studies, although most studies examined current and former smokers. In addition, this study targeted current smokers, as previous smokers represent a wide range of smoking behaviors that are difficult to interpret in analysis. LOS was 0.5 days longer for the diabetic subjects versus non-diabetic subjects, and 0.3 days longer for males than females, but did not differ for smokers versus non-smokers (data not shown). Additionally, downstream costs differed by $<\$ 60$ between the diabetic/nondiabetic groups, smoking/non-smoking groups, and sexes.

Low-risk patients were more likely to have an outpatient visit, while high-risk patients were more likely to have an inpatient visit; however, neither group was more likely to have repeat non-invasive CV testing. Many studies use risk categories as inclusion or stratification techniques, but this study suggests that it may be more useful to look at this variable as a covariate rather than inclusion or stratification. However, a larger issue might be the lack of a standardized risk measurement for $\mathrm{CV}$ studies. 
While standardized coronary risk scores, such as the Framingham Coronary Heart Disease Risk Score [14], and coronary risk for patients with comorbidities, such as the Atherosclerotic Cardiovascular Disease (ASCVD) Risk Assessment Tool [15], exist for clinical care, there are issues with their use in research. In addition to the possible lack of availability in EMRs, which limits use in retrospective studies, risk scores often include key variables within their computation that prohibits the use of the variable in analysis. For example, many risk scores include age in the risk score computation; therefore, if age is included as an independent variable in the analysis, it is counted twice, thus presenting issues for analysis. Factors such as age and comorbidities often play an important role in health outcomes, and their contribution to the outcome of interest might need to be assessed as a variable rather than as part of an index variable or composite score.

Finally, many risk scores calculate the risk of a cardiac event 10 or more years after the index event, which may not be a useful timeframe when considering immediate outcomes. We sought to address these issues by using a risk stratification that is not dependent on any other potential covariate and is more temporally related to the outcome of interest.

The purpose of this study was not to build a predictive model but rather to assess the contribution of testing modalities to downstream HCRU while controlling for potential covariates. However, the $R^{2}$ (coefficient of determination) for the regression models, which measures the overall fit of the model and ranges from 0.0 to 1.0 , with 1.0 indicating a perfect fit, ranged from 0.01 to 0.07 , indicating that key covariates may not be included in these models.

As with all retrospective studies, we were limited by the quality of the available data. It is well known that use of retrospective data has several limitations, such as inconsistent measuring and reporting in the source material. In this study, we systematically abstracted data via a database query to reduce errors at this phase, sought to validate data from two sources wherever possible, and thoroughly examined the data for inconsistencies prior to analysis to reduce these issues. However, we were examining downstream costs and revisits and cannot be certain that the patients in our study did not seek additional healthcare outside of the study's healthcare system. In this case, our reporting would underrepresent downstream costs. In this study, omitted variable bias is a potential limitation as there are many clinical presentation variables not accounted for. Additional variables that have yet to be identified could also influence the model fit and ultimately influence the individual contribution of the testing modality on the outcome. In this study, we added a selection of covariates deemed appropriate by a panel of cardiology physicians and nurses, as well as researchers with cardiology experience, in order to reduce the effect of this potential bias.

\section{Conclusions}

Although the selection of which non-invasive CV testing is the best fit for a particular patient ultimately relies on clinical decision making and physician discretion, this study provides evidence that SPECT-MPI does not result in more downstream HCRU than ETT or ECHO. While initial costs may be higher, SPECT-MPI may present an advantage as it was associated with a lower likelihood of repeat non-invasive CV testing and inpatient visits.

Acknowledgements The authors acknowledge Dr. Warren Skea and Todd Evans for their contribution to the development of the project, and also thank Laurie Orloski, PharmD, for providing writing support through Xcenda, Inc.

Author Contributions DV and WTS contributed to and were responsible for study design, data collection and cleaning, analysis and interpretation, and manuscript preparation. JRS, TMK, RMK and CRH contributed to the study design and manuscript preparation.

\section{Compliance with Ethical Standards}

Funding Funding for this project was provided by Astellas Pharma Global Development, Inc.

Data Sharing Institutional practices prohibit data sharing.

Conflict of interest James R. Spalding, Therese M. Kitt, and Rita M. Kristy are employees of Astellas Pharma Global Development Inc. Cristy R. Houle was an employee of Astellas Pharma Global Development Inc.during the project. Dana Villines reports funding for this project from Astellas Pharma Inc., although analysis was performed at another institution and was independent of the funding source. Thomas Summerfelt received funding from Astellas Pharma Inc. to collect, analyze, and participate in the collaborative project.

Open Access This article is distributed under the terms of the Creative Commons Attribution-NonCommercial 4.0 International License (http://creativecommons.org/licenses/by-nc/4.0/), which permits any noncommercial use, distribution, and reproduction in any medium, provided you give appropriate credit to the original author(s) and the source, provide a link to the Creative Commons license, and indicate if changes were made.

\section{References}

1. Heidenreich PA, Trogdon JG, Khavjou OA, Butler J, Dracup K, Ezekowitz MD, et al. Forecasting the future of cardiovascular disease in the United States: a policy statement from the American Heart Association. Circulation. 2011;123(8):933-44.

2. Skelly AC, Hashimoto R, Buckley DI, Brodt ED, Noelck N, Totten AM, et al. Noninvasive testing for coronary artery disease. Rockville: AHRQ Comparative Effectiveness Reviews; 2016.

3. Rybicki FJ, Udelson JE, Peacock WF, Goldhaber SZ, Isselbacher EM, Kazerooni E, et al. 2015 ACR/ACC/AHA/AATS/ACEP/ ASNC/NASCI/SAEM/SCCT/SCMR/SCPC/SNMMI/STR/STS appropriate utilization of cardiovascular imaging in emergency department patients with chest pain: a joint document of the 
American College of Radiology Appropriateness Criteria Committee and the American College of Cardiology Appropriate Use Criteria Task Force. J Am Coll Cardiol. 2016;67(7):853-79.

4. Bittencourt MS, Hulten EA, Murthy VL, Cheezum M, Rochitte $\mathrm{CE}$, Di Carli MF, et al. Clinical outcomes after evaluation of stable chest pain by coronary computed tomographic angiography versus usual care: a meta-analysis. Circ Cardiovasc Imaging. 2016;9(4):e004419.

5. Hulten E, Pickett C, Bittencourt MS, Villines TC, Petrillo S, Di Carli MF, et al. Outcomes after coronary computed tomography angiography in the emergency department: a systematic review and meta-analysis of randomized, controlled trials. J Am Coll Cardiol. 2013;61(8):880-92.

6. Hulten EA, Carbonaro S, Petrillo SP, Mitchell JD, Villines TC. Prognostic value of cardiac computed tomography angiography: a systematic review and meta-analysis. J Am Coll Cardiol. 2011;57(10):1237-47.

7. Makani H, Bangalore S, Halpern D, Makwana HG, Chaudhry FA. Cardiac outcomes with submaximal normal stress echocardiography: a meta-analysis. J Am Coll Cardiol. 2012;60(15):1393-401.

8. Nielsen LH, Ortner N, Norgaard BL, Achenbach S, Leipsic J, Abdulla J. The diagnostic accuracy and outcomes after coronary computed tomography angiography vs. conventional functional testing in patients with stable angina pectoris: a systematic review and meta-analysis. Eur Heart J Cardiovasc Imaging. 2014;15(9):961-71.

9. Hachamovitch R, Berman DS, Kiat H, Cohen I, Friedman JD, Shaw LJ. Value of stress myocardial perfusion single-photon emission computed tomography in patients with normal resting electrocardiograms: an evaluation of incremental prognostic value and cost-effectiveness. Circulation. 2002;105(7):823-9.

10. Hayashino Y, Nagata-Kobayashi S, Morimoto T, Maeda K, Shimbo T, Fukui T. Cost-effectiveness of screening for coronary artery disease in asymptomatic patients with Type 2 diabetes and additional atherogenic risk factors. J Gen Intern Med. 2004;19(12):1181-91.

11. Kuntz KM, Fleischmann KE, Hunink MG, Douglas PS. Costeffectiveness of diagnostic strategies for patients with chest pain. Ann Intern Med. 1999;130(9):709-18.

12. Lee DS, Jang MJ, Cheon GJ, Chung JK, Lee MC. Comparison of the cost-effectiveness of stress myocardial SPECT and stress echocardiography in suspected coronary artery disease considering the prognostic value of false-negative results. J Nucl Cardiol. 2002;9(5):515-22.

13. Thygesen K, Alpert JS, Jaffe AS, Simoons ML, Chaitman BR, White HD, et al. Third universal definition of myocardial infarction. J Am Coll Cardiol. 2012;60(16):1581-98.

14. Wilson PW, D'Agostino RB, Levy D, Belanger AM, Silbershatz H, Kannel WB. Prediction of coronary heart disease using risk factor categories. Circulation. 1998;97(18):1837-47.

15. Goff DC Jr, Lloyd-Jones DM, Bennett G, Coady S, D'Agostino RB Sr, Gibbons R. et al. 2013 ACC/AHA guideline on the assessment of cardiovascular risk: a report of the American College of Cardiology/American Heart Association Task Force on Practice Guidelines. J Am Coll Cardiol. 2014;63(25 Pt B):2935-59. 